\title{
Atrial myxoma producing a saddle embolus in a child
}

\author{
M.A.R. KHAN and M.A. MUJAHED \\ Departments of Paediatrics and Thoracic Surgery, Victoria Hospital, Blackpool
}

\begin{abstract}
Atrial myxomata are rare in childhood. A case is described in a boy aged 7 years. The presenting feature was a saddle embolus, requiring immediate surgical intervention. Further investigations showed that a large part of the myxoma remained in the atrium; this portion was successfully removed. This mode of presentation is unusual. The presence of calcification within the myxoma was detectable on intensifier screen examination. The biochemical abnormalities frequently associated with myxomata were not found. The importance of recognizing a myxoma as the cause of a saddle embolus in an otherwise healthy child is emphasized and a number of unusual features of the present case are described.
\end{abstract}

\section{CASE REPORT}

A 7-year-old boy was admitted to Victoria Hospital, Blackpool, on 1 December 1969 as an emergency. He had developed increasingly painful feet the day before admission. He had vomited several times and passed blood and mucus with rather loose motions. There was no history of any previous illness.

Physical examination showed a thin boy with slight dehydration. He was apyrexial. The pulse was regular with a rate of 140 per minute; the blood pressure in the right arm was $90 \mathrm{~mm}$. $\mathrm{Hg}$ systolic. Both the femoral and the more distant pulses were absent. Pulsations in the abdominal aorta were palpable. Both lower extremities were cold and slightly cyanosed below the level of the iliac crests. The heart was clinically normal apart from the presence of a highpitched systolic murmur internal to the apex beat. Knee jerks were diminished and ankle jerks were absent. Plantar responses were flexor. Rectal examination showed blood and mucus on the glove finger.

INVESTIGATIONS $\mathrm{Hb} \quad 13.4 \mathrm{~g} . / 100 \mathrm{ml}$, white cells $8,800 / \mathrm{cu}$. mm. with a normal differential count, ESR $25 \mathrm{~mm}$. per hour (Westergren). Sodium $134 \mathrm{mEq} / 1$, potassium $5.7 \mathrm{mEq} / \mathrm{l}$, blood urea $85 \mathrm{mg} . / 100 \mathrm{ml}$., plasma proteins: albumin $3.4 \mathrm{~g} . / 100 \mathrm{ml}$, globulin 2.4 g. $/ 100 \mathrm{ml}$., electrophoresis normal. ASOT 125 units $/ \mathrm{ml}$. Cold antibodies absent. Chest radiograph normal. ECG slight left ventricular preponderance and sinus rhythm.

In view of the fact that this was a child presenting as a saddle embolus, who also had a distinct murmur in the mitral area, a likely diagnosis was considered to be an atrial myxoma. There was no evidence or history of other embolus.

Because of the ischaemic changes in the legs, the abdominal aorta was explored immediately through a long left paramedian incision. There was some dis- colouration of the pelvic colon. Aortic pulsation was present as far as 1.5 in. ( $38 \mathrm{~mm}$.) above the bifurcation. Both common iliac arteries and aorta were clamped; the embolus, which was rather friable and gelatinous, was removed in its entirety (Fig. 1). Following this procedure the circulation in the lower extremities and the pelvic colon was restored promptly and recovery was uneventful. Histologically, the specimen removed $(3 \times 1.7 \times 1.5 \mathrm{~cm}$.) was found to be composed of myxomatous stroma with scattered stellate cells. An unusual feature was the presence of central calcifications.

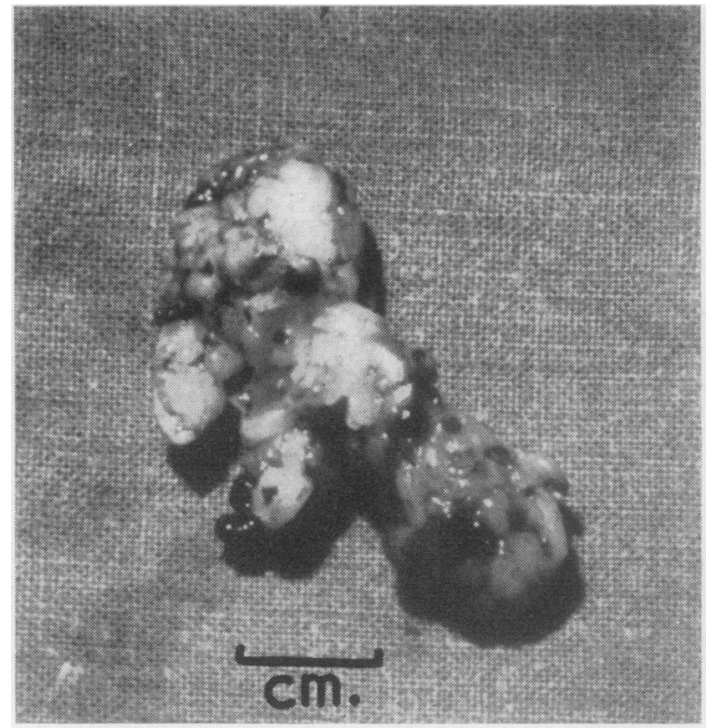

FIG. 1. Portion of the atrial myxoma removed from the aortic bifurcation. 
Detailed haemodynamic and angiocardiographic studies were carried out 10 days after embolectomy. On the image intensifier small intra-atrial calcium deposits were clearly visible. The right heart appeared normal. The left heart was opacified by contrast medium injected into the outflow tract of the right ventricle. A large tumour filling defect, within an otherwise normal left atrium, was clearly demonstrated in the cine-radiograph, there being a pronounced movement of the tumour mass towards the mitral orifice in atrial systole. This large tumour defect was also demonstrated in a large film angiogram series, but was less clearly identifiable than in motion pictures. Figure 2 is a print of one of the large film series with enhancement of contrast by means of subtraction radiography.

With the diagnosis confirmed, the left atrium was explored on cardiopulmonary bypass. There was a large, well pedunculated gelatinous tumour, filling nearly the whole of the left atrial chamber. The stalk arose from the posterior atrial wall just below and medial to the right inferior pulmonary vein. The mitral valve appeared normal. The tumour was excised, including the atrial wall around its base.

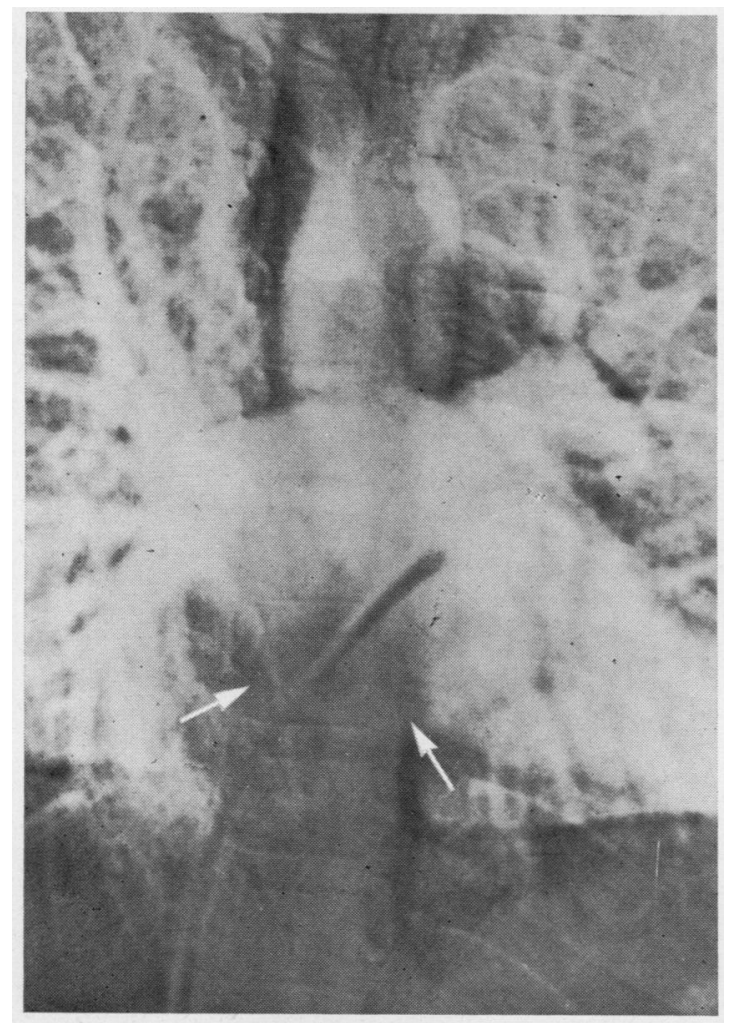

FIG. 2. Antero-posterior large film angiogram with enhancement of contrast by means of subtraction radiography. There is a large filling defect in the left atrium.
Post-operative recovery was uneventful and the child was discharged two weeks later. A pansystolic murmur persisted at the apex ; this was thought to be due to damage to the mitral valve caused by the atrial myxoma, the 'wrecking ball effect' referred to by Harvey (1968).

Histologically the appearances were similar to those of the embolectomy specimen. The tumour appeared to arise from endocardium, but the deeper layers of this and the myocardium were intact. There was a distinct margin of normal tissue at the base. Some calcification was present in the stroma of the tumour close to its base. There was no evidence of malignancy.

\section{DISCUSSION}

About $35 \%$ of primary intra-cardiac tumours are myxomata (Wood, 1956). In only 9 of the 200 cases collected by Cumming and Finkel (1961) was the tumour found in children. Half of these children presented with systemic emboli, predominantly cerebral with frequently a fatal outcome. Only one presented as saddle embolus of the abdominal aorta. The joint research study of the Association of European Cardiologists has collected a total of 22 primary cardiac tumours in the past five years. Three of these tumours were myxomata and only one presented as a femoral artery embolus (van der Hauwaert, 1970). Early diagnosis of the tumour is important in view of the poor prognosis in unrecognized cases.

This case illustrates the association of atrial myxoma with saddle embolus. Arterial emboli in children are extremely rare in the absence of an intra-cardiac shunt or bacterial endocarditis. They occur occasionally in endocardial fibroelastosis and the cardiomyopathies. The history and findings in this child did not suggest the presence of any of the above conditions. The clinical findings were non-specific apart from the obvious presence of a saddle embolus. In order to restore the vascular supply to the legs urgent embolectomy was essential. For satisfactory removal of the embolus, the abdominal approach was used in preference to bilateral femoral arteriotomy.

Myxomata may occur simultaneously in right and left atria (Frankenfeld, Waters, and Steiner, 1960 ; Cumming and Finkel, 1961). Therefore it is essential to carry out right and left heart studies. The diagnosis of these tumours may be made by the identification of a filling defect within a contrast-filled atrium. A large tumour mass, such as that present in the case described, can be identified in good quality large film angiograms as a filling defect which may vary in position with variation in the cardiac phase at the moment of film 
exposure, but the method of choice is cineradiography. Multiple projections may have to be used, and even so there may be difficulty in reaching a firm diagnosis of a very small intra-cavitary pedunculated tumour mass.

The presenting symptoms in this case were similar to those in a child described by Young and Hunter (1947), in that the child presented with ischaemic changes in the legs and a bowel disturbance. It seems likely that a frond of the tumour had obstructed the inferior mesenteric artery. This mode of presentation is rare, as the great majority of recognized emboli from atrial myxomata are cerebral. It may be that the large size of the embolus prevented its entering the branches of the arch of the aorta. In adults atrial myxomata have presented as saddle emboli in several reported cases. Usually the obstruction to blood flow to the legs has been recognized, but in otherwise apparently healthy adults the sudden development of ischaemia of the legs has been difficult to interpret and delay in treatment has frequently led to death (Brewin, 1951 ; Edwards and Johnson, 1959; Carter, Lowe, and Hill, 1960). It is of interest that the presenting symptom in several of these cases was increasingly severe pain in the feet.

Detailed questioning of both the child and the parents failed to produce evidence of preceding symptoms, which is unusual as most patients experience dyspnoea on exertion.

This tumour arose from the posterior wall of the left atrium, rather than from the usual site which is the interatrial septum. It contained foci of calcification revealed by intensifier screening examination.

In view of the possibility of recurrence of the tumour, as described by Bahl, Oliver, Ferguson, Schad, and Parker (1969) a margin of healthy atrium was excised at the base of the tumour.

We are grateful to Mr. J. S. Glennie and Dr. J. P. Bound for their permission to report this case. We are indebted to Dr. K. A. Rowley for the angiocardiographic interpretations, and to Dr. D. S. Harry for his review of the sections of the tumour. We thank Dr. W. F. W. E. Logan for the haemodynamic and angiocardiographic investigations, and for his helpful suggestions in the preparation of this paper.

\section{REFERENCES}

Bahl, O. P., Oliver, G. C., Ferguson, T. B., Schad, N., and Parker, B. M. (1969). Recurrent left atrial myxoma. Circulation, 40, 673. Brewin, T. B. (1951). 'Myxoma' of the heart. Guy's Hosp. Rep., $100,278$.

Carter, A. B., Lowe, K. G., and Hill, I. G. W. (1960). Cardiac myxomata and aortic saddle embolism. Brit. Heart J., 22, 502.

Cumming, G. R., and Finkel, K. (1961). Intracardiac myxoma involving the right and left atria in a young patient. J. Pediat., 58, 559.

Edwards, A. T., and Johnson, W. (1959). A case of myxoma of the left atrium with peripheral arterial emboli. Brit. J. Surg., 46, 371 .

Frankenfeld, R. H., Waters, C. H., and Steiner, R. C. (1960). Bilateral myxomas of the heart. Ann. intern. Med., 53, 827.

Harvey, W. P. (1968). Clinical aspects of cardiac tumors. Amer. J. Cardiol., 21, 328.

van der Hauwaert, Luc (1970). Cardiac tumours in infancy and childhood. Proceedings of the Association of European Paediatric Cardiologists, VI, 31.

Wood, P. (1956). Diseases of the Heart and Circulation, 2nd ed. Eyre and Spottiswoode, London.

Young, R. D., and Hunter, W. C. (1947). Primary myxoma of the left ventricle with embolic occlusion of the abdominal aorta and renal arteries. Arch. Path., 43, 86. 